\title{
A SHORT NOTE ON ENHANCED DENSITY SETS
}

\author{
SILVANO DELLADIO \\ Department of Mathematics, University of Trento, via Sommarive 14, Povo, 38123 Trento, Italy \\ e-mail:delladio@science.unitn.it
}

(Received 5 December 2009; revised 1 July 2010; accepted 11 April 2011)

\begin{abstract}
We give a simple proof of a statement extending Fu's (J.H.G. Fu, Erratum to 'some remarks on legendrian rectiable currents', Manuscripta Math. 113(3) (2004), 397-401) result: 'If $\Omega$ is a set of locally finite perimeter in $\mathbb{R}^{2}$, then there is no function $f \in C^{1}\left(\mathbb{R}^{2}\right)$ such that $\nabla f\left(x_{1}, x_{2}\right)=\left(x_{2}, 0\right)$ at a.e. $\left(x_{1}, x_{2}\right) \in \Omega^{\prime}$. We also prove that every measurable set can be approximated arbitrarily closely in $L^{1}$ by subsets that do not contain enhanced density points. Finally, we provide a new proof of a Poincaré-type lemma for locally finite perimeter sets, which was first stated by Delladio (S. Delladio, Functions of class $C^{1}$ subject to a Legendre condition in an enhanced density set, to appear in Rev. Mat. Iberoamericana).
\end{abstract}

2010 Mathematics Subject Classification. Primary 28Axx, 28A75, 49Q15; Secondary 26A45.

1. Introduction. In [3] we introduced the notions of enhanced density point and enhanced density set.

Definition 1.1. Let $\Omega$ be a measurable subset of $\mathbb{R}^{n}$. Then $x \in \mathbb{R}^{n}$ is said to be a ' point of enhanced density of $\Omega$ ' if

$$
\lim _{r \downarrow 0} \frac{\mathcal{L}^{n}(B(x, r) \backslash \Omega)}{r^{n+1}}=0 .
$$

By $\Omega_{\bullet}$ we denote the set of all the points of enhanced density of $\Omega$. We say that ' $\Omega$ is an enhanced density set' whenever $\mathcal{L}^{n}\left(\Omega \backslash \Omega_{\bullet}\right)=0$.

The family of enhanced density sets includes locally finite perimeter sets (we refer the reader to [2, Section 3.3] or [5, Definition 7.5.4] for the definition). In fact, even stronger density property proved by Delladio [3] holds.

THEOREM 1.1. Let $\Omega$ be a locally finite perimeter subset of $\mathbb{R}^{n}$. Then

$$
\lim _{r \downarrow 0} \frac{\mathcal{L}^{n}(B(x, r) \backslash \Omega)}{r^{n+\frac{n}{n-1}}}=0
$$

at a.e. $x \in \Omega$. In particular, $\Omega$ is an enhanced density set.

The following result, given in [3], provides a generalisation of the classical Poincare's Lemma. 
THEOREM 1.2. Let $\lambda$ and $\mu$ be differential forms of class $C^{1}$ in $\mathbb{R}^{n}$, respectively, of degree $h$ and $h+1$ (with $h \geq 0)$. If

$$
K:=\left\{x \in \mathbb{R}^{n} \mid d \lambda(x)=\mu(x)\right\},
$$

then $K_{\bullet} \subset K$ and $\left.(d \mu)\right|_{K_{\bullet}}=0$.

REMARK 1.1. Let $f \in C^{1}\left(\mathbb{R}^{n}\right), g \in C^{1}\left(\mathbb{R}^{n}, \mathbb{R}^{n}\right)$ and

$$
K:=\left\{x \in \mathbb{R}^{n} \mid \nabla f(x)=g(x)\right\} .
$$

If we apply Theorem 1.2 with

$$
\lambda:=f, \quad \mu:=\sum_{j=1}^{n} g_{j} d x_{j}
$$

and observe that

$$
K=\left\{x \in \mathbb{R}^{n} \mid d \lambda(x)=\mu(x)\right\}, \quad d \mu=\sum_{\substack{i, j=1 \\ i<j}}^{n}\left(\frac{\partial g_{j}}{\partial x_{i}}-\frac{\partial g_{i}}{\partial x_{j}}\right) d x_{i} \wedge d x_{j},
$$

then we obtain $K_{\bullet} \subset K$ and

$$
\left.(\operatorname{curl} g)\right|_{K_{\bullet}}=0
$$

where curl is defined as in [1].

This note collects the following three independent results related to enhanced density sets.

- The first one extends [4, Corollary 2], which states, 'If $\Omega$ is a set of locally finite perimeter in $\mathbb{R}^{2}$, then there is no function $f \in C^{1}\left(\mathbb{R}^{2}\right)$ such that $\nabla f\left(x_{1}, x_{2}\right)=\left(x_{2}, 0\right)$ at a.e. $\left(x_{1}, x_{2}\right) \in \Omega^{\prime}$. Observe that this fact, proved by $\mathrm{Fu}$ [4] through a quite technical argument based on integral currents, is an immediate and GMT-free consequence of Remark 1.1 and Theorem 1.1.

- The second one states that every measurable set can be approximated arbitrarily closely in $L^{1}$ by subsets that do not contain enhanced density points.

- The third one is a direct proof, using nothing but Stokes for Whitney's flat chains, of a Poincaré-type lemma for locally finite perimeter sets.

\section{Statements and proofs of the results}

\subsection{Generalisation of $[4$, Corollary 2]}

THEOREM 1.3. Let $\Omega$ be an enhanced density set, e.g. a locally finite perimeter subset of $\mathbb{R}^{n}$. Let $\mu$ be a differential form of class $C^{1}$ in $\mathbb{R}^{n}$ (and degree $h \geq 1$ ) and assume that

$$
\mathcal{L}^{n}(\Omega \cap E)>0, \quad E:=\left\{x \in \mathbb{R}^{n} \mid d \mu(x) \neq 0\right\} .
$$

Then there is no differential form of class $C^{1}$ in $\mathbb{R}^{n}$ (and degree $h-1$ ) such that $d \lambda=\mu$ a.e. in $\Omega \cap E$. 
We will obtain Theorem 1.3 as a trivial corollary of Theorem 1.4 below. In order to prove the latter, we state a simple proposition.

Proposition 1.1. The following facts hold:

(i) Let $\Omega$ and $\Omega$ ' be measurable subsets of $\mathbb{R}^{n}$ such that ' $\Omega$ is a subset of $\Omega$ ' in measure', i.e. $\mathcal{L}^{n}\left(\Omega \backslash \Omega^{\prime}\right)=0$. Then one has $\Omega_{\bullet} \subset \Omega_{\text {. }}^{\prime}$.

(ii) Let $\Omega$ and $A$ be, respectively, a measurable subset of $\mathbb{R}^{n}$ and an open subset of $\mathbb{R}^{n}$. Then $\Omega_{\bullet} \cap A \subset(\Omega \cap A)$. holds, while the opposite inclusion is false in general.

Proof. The assertion (i) is obvious. As for (ii), let $x \in \Omega_{\bullet} \cap A$ and observe that since $A$ is open, then

$$
B(x, r) \backslash(\Omega \cap A)=(B(x, r) \backslash \Omega) \cup(B(x, r) \backslash A)=B(x, r) \backslash \Omega
$$

provided $r$ is small enough. Hence,

$$
\lim _{r \downarrow 0} \frac{\mathcal{L}^{n}(B(x, r) \backslash(\Omega \cap A))}{r^{n+1}}=\lim _{r \downarrow 0} \frac{\mathcal{L}^{n}(B(x, r) \backslash \Omega)}{r^{n+1}}=1,
$$

namely $x \in(\Omega \cap A)$. The assertion about the opposite inclusion is proved by the following example, where we assume $n=2$. Let

$$
\Omega:=\mathbb{R}^{2} \backslash\{(0,0)\}, \quad A:=\Omega .
$$

Then one has

$$
\Omega_{\bullet} \cap A=A, \quad(\Omega \cap A)_{\bullet}=\mathbb{R}^{2} .
$$

THEOREM 1.4. Let $\Omega$ be a measurable subset of $\mathbb{R}^{n}, \mu$ be a differential form of class $C^{1}$ in $\mathbb{R}^{n}$ (and degree $h \geq 1$ ) and assume that

$$
\mathcal{L}^{n}(\Omega \cap E)>0, \quad E:=\left\{x \in \mathbb{R}^{n} \mid d \mu(x) \neq 0\right\} .
$$

If there exists a differential form $\lambda$ of class $C^{1}$ in $\mathbb{R}^{n}$ (and degree $\left.h-1\right)$ such that $d \lambda=\mu$ a.e. in $\Omega \cap E$, then $\Omega$ is not an enhanced density set.

Proof. Let $K$ be defined as in Theorem 1.2. Since $E$ is open, it follows from Proposition 1.1 that

$$
\Omega_{\bullet} \cap E \subset(\Omega \cap E)_{\bullet} \subset K_{\bullet} .
$$

Then the set $\Omega_{\bullet} \cap E$ has to be empty, by Theorem 1.2. Hence,

$$
\mathcal{L}^{n}\left(\Omega \backslash \Omega_{\bullet}\right) \geq \mathcal{L}^{n}\left((\Omega \cap E) \backslash\left(\Omega_{\bullet} \cap E\right)\right)=\mathcal{L}^{n}(\Omega \cap E)>0,
$$

namely $\Omega$ is not an enhanced density set.

\subsection{Approximation by sets without enhanced density points}

THEOREM 1.5. Let $\varepsilon>0$ be fixed arbitrarily. Then there exists an open subset $A$ of $\mathbb{R}^{n}$ such that $\mathcal{L}^{n}(A) \leq \varepsilon$ and $(\Omega \backslash A)$. is empty for all measurable subsets $\Omega$ of $\mathbb{R}^{n}$. 
Proof. Let $g \in C^{1}\left(\mathbb{R}^{n}, \mathbb{R}^{n}\right)$ be such that $\operatorname{curl} g(x) \neq 0$ at all $x \in \mathbb{R}^{n}$. Consider the open subsets of $\mathbb{R}^{n}$

$$
\Gamma_{0}:=\{|x|<1\}, \quad \Gamma_{j}:=\{j<|x|<j+1\} \quad(j=1,2, \ldots) .
$$

Then, for all $j$ we can find

(i) an open neighbourhood $A_{j}^{\prime}$ of $\partial \Gamma_{j}$ such that

$$
\mathcal{L}^{n}\left(A_{j}^{\prime}\right) \leq \frac{\varepsilon}{2^{j+2}}
$$

(ii) an open subset $A_{j}^{\prime \prime}$ of $\Gamma_{j}$ and a function $f_{j} \in C_{0}^{1}\left(\Gamma_{j}\right)$ such that

$$
\mathcal{L}^{n}\left(A_{j}^{\prime \prime}\right) \leq \frac{\varepsilon}{2^{j+2}}
$$

and

$$
\left.\left(\nabla f_{j}\right)\right|_{\Gamma_{j} \backslash A_{j}^{\prime \prime}}=\left.g\right|_{\Gamma_{j} \backslash A_{j}^{\prime \prime}}
$$

by [1, Theorem 1].

From $(1.1)$ and Remark 1.1, it follows that (for $j=0,1, \ldots$ ) there are no points of enhanced density of the set

$$
R_{j}:=\Gamma_{j} \backslash\left(A_{j}^{\prime} \cup A_{j}^{\prime \prime}\right) \subset \Gamma_{j} \backslash A_{j}^{\prime \prime} .
$$

Since $R_{j} \subset \subset \Gamma_{j}$ for all $j$, there are no points of enhanced density of

$$
\bigcup_{j=0}^{\infty} R_{j}=\bigcup_{j=0}^{\infty} \Gamma_{j} \backslash\left(A_{j}^{\prime} \cup A_{j}^{\prime \prime}\right)=\mathbb{R}^{n} \backslash A,
$$

where $A$ is the open set defined by

$$
A:=\bigcup_{j=0}^{\infty}\left(A_{j}^{\prime} \cup A_{j}^{\prime \prime}\right) .
$$

The conclusion follows from Proposition 1.1(i) and by observing that

$$
\mathcal{L}^{n}(A) \leq \sum_{j=0}^{\infty}\left[\mathcal{L}\left(A_{j}^{\prime}\right)+\mathcal{L}\left(A_{j}^{\prime \prime}\right)\right] \leq \sum_{j=0}^{\infty} \frac{\varepsilon}{2^{j+1}}=\varepsilon .
$$

2.3. A Poincaré-type lemma for locally finite perimeter sets. The following fact is an immediate consequence of Theorems 1.1 and 1.2. It has first been stated in [3]. Here we give an alternative short proof using nothing but Stokes for Whitney's flat chains [6]. This new proof is based on a global argument that could reveal to be useful for treating similar issues in the context of integral currents.

THEOREM 1.6. Let $\lambda$ and $\mu$ be $C^{1}$ forms of degree $h$ and $h+1$, respectively, with $0 \leq h \leq n-2$. Assume that $d \lambda=\mu$ almost everywhere in a locally finite perimeter set $\Omega$. Then one also has $d \mu=0$ almost everywhere in $\Omega$. 
Proof. Let $\omega$ be any smooth form of degree $n-h-2$ with compact support. Then one has (adopting the notation of [5, Section 7.2])

$$
\begin{aligned}
\int_{\Omega} d \mu \wedge \omega & =\int_{\Omega} d(\mu \wedge \omega)+(-1)^{h} \int_{\Omega} \mu \wedge d \omega \\
& =\partial \llbracket \Omega \rrbracket(\mu \wedge \omega)+(-1)^{h} \int_{\Omega} \mu \wedge d \omega \\
& =\partial \llbracket \Omega \rrbracket(d \lambda \wedge \omega)+(-1)^{h} \int_{\Omega} \mu \wedge d \omega \\
& =\partial \llbracket \Omega \rrbracket(d(\lambda \wedge \omega))+(-1)^{h+1} \partial \llbracket \Omega \rrbracket(\lambda \wedge d \omega)+(-1)^{h} \int_{\Omega} \mu \wedge d \omega .
\end{aligned}
$$

Now, by [5, Remark 7.5.6 and Theorem 7.9.2], a locally finite perimeter set is a flat chain in the sense of Whitney. We get

$$
\partial \llbracket \Omega \rrbracket(d(\lambda \wedge \omega))=0
$$

by [6, Chapter V, Section 3]. Hence,

$$
\begin{aligned}
\int_{\Omega} d \mu \wedge \omega & =(-1)^{h+1} \int_{\Omega} d(\lambda \wedge d \omega)+(-1)^{h} \int_{\Omega} \mu \wedge d \omega \\
& =-(-1)^{h} \int_{\Omega} d \lambda \wedge d \omega+(-1)^{h} \int_{\Omega} \mu \wedge d \omega \\
& =0 .
\end{aligned}
$$

The conclusion follows from the arbitrariness of $\omega$.

\section{REFERENCES}

1. G. Alberti, A Lusin-type theorem for gradients, J. Funct. Anal. 100 (1991), 110-118.

2. L. Ambrosio, N. Fusco and D. Pallara, Functions of bounded variation and free discontinuity problems, Oxford mathematical monographs (Oxford University Press, Oxford, UK, 2000).

3. S. Delladio, Functions of class $C^{1}$ subject to a Legendre condition in an enhanced density set, to appear on Rev. Mat. Iberoamericana.

4. J. H. G. Fu, Erratum to 'some remarks on legendrian rectifiable currents', Manuscripta Math. 113(3) (2004), 397-401. 2008).

5. S. G. Krantz and H. R. Parks, Geometric integration theory (Birkhäuser, Boston, MA, 1957).

6. H. Whitney, Geometric integration theory (Princeton University Press, Princeton, NJ, 